\title{
Management strategies of enterovirus D68 outbreaks: current perspectives
}

This article was published in the following Dove Press journal: Virus Adaptation and Treatment

\section{Natacha Milhano \\ Kaja Sverdrup Borge \\ Karoline Bragstad \\ Susanne G Dudman}

Domain for Environmental Health and Infectious Disease Control, Norwegian Institute of Public Health, Oslo, Norway
Correspondence: Natacha Milhano Domain for Environmental Health and Infectious Disease Control, Norwegian Institute of Public Health, Lovisenberggata 8, 0403 Oslo, Norway

Tel +47 21076734

Email natmil66@gmail.com

\begin{abstract}
Following its discovery in California in 1962, enterovirus D68 (EV-D68) was reported only sporadically around the world. In August 2014, a marked increase of EV-D68 cases in young children with severe respiratory infections was reported in the USA and Canada and later in Europe and Asia. Some of these cases were also found to be associated with acute flaccid paralysis, which exacerbated public health concern, and has since triggered international efforts to strengthen both EV-D68 and acute flaccid paralysis surveillance systems. This review summarizes the current knowledge on EV-D68, offering an overview of EV-D68 epidemiology, clinical presentations, diagnostic methodologies, and treatment strategies, as well as surveillance and outbreak management.
\end{abstract}

Keywords: enterovirus D68, AFP, diagnostics, treatment, surveillance, outbreak

\section{Introduction}

Human enteroviruses (EVs) are responsible for a wide variety of human infections, ranging from mild, nonspecific illness to severe diseases, such as hand, foot, and mouth disease, respiratory infections, herpangina, and encephalitis, among others. Enterovirus D68 (EV-D68), an acid-labile EV belonging to EV group D, is mainly associated with respiratory disease and was identified for the first time in hospitalized children presenting with pneumonia and bronchiolitis in California in $1962 .{ }^{1}$ In the following years, this virus was sporadically reported until 2008, when cases of EV-D68 were detected in the Philippines, Japan, the Netherlands, and the USA. ${ }^{2}$ The majority of the detections were among young children under 4 years of age, presenting with respiratory complications and requiring hospitalization. ${ }^{3,4}$ Starting from August 2014, an increase in the number of EV-D68 cases was initially reported in the USA, followed by Canada and Europe. This marked increase in EV-D68 cases, many of which were associated with neurological complications, has since triggered international public health concern and set in motion recommendations for worldwide increased surveillance for EV-D68. ${ }^{2,5}$

This review summarizes the current knowledge on EV-D68, including an overview of EV-D68 surveillance and outbreak management strategies. With this purpose, a PubMed database search, limited to publications from the last 10 years, was performed using EV-D68, EV-D68 AND drug therapy, EV-D68 AND drug effects, EV-D68 AND pathogenicity, and EV-D68 AND vaccine as keywords. This search resulted in 238 records, for which the abstracts were screened based on a set of inclusion and exclusion criteria. These included EV-D68 epidemiology, clinical presentation, surveillance, and outbreak management and excluded case reports, modeling, and environmental 
studies. As a result of this screening, 78 publications were systematically reviewed, of which 59 were included for the current review.

\section{Epidemiology}

The increase in EV-D68 cases reported in young children in the summer of 2014 triggered an alarm in the international public health community. It was soon after revealed that an unprecedented high number of EV-D68 cases also presented with acute flaccid paralysis (AFP), namely 120 cases in the USA and another three in Europe, two of which were in Norway and one in France..$^{6-8}$ In view of these detections and the increasing evidence of a potential association of EV-D68 with AFP, the European Centre for Disease Prevention and Control advised European Union (EU)/ European Economic Area (EEA) countries to strengthen respiratory sample screening for EVs and EV typing and to strengthen AFP surveillance as well as EV surveillance, with a particular focus on cases with affected central nervous system function. ${ }^{5}$ These measures envisaged enhancing polio surveillance and allowed for the detection of unusual clusters of neurological disease or trends among nonpolio AFP cases. For this purpose, new diagnostic methods were developed specifically for detection of EV-D68, and several retrospective studies ensued, showing an increase in EV-D68 cases in preceding years, many of which were considered at the time of detection as rhinovirus (RV) infections. ${ }^{9,10}$ Retrospective studies in the Netherlands showed an increased circulation of the virus in the country from 2010 to 2014. . $^{3,11}$

In the following year, 2015, few or no EV-D68 cases were reported, after which an upsurge of cases re-occurred in 2016, both in Europe and in the USA. ${ }^{12,13}$ During the summer of 2016, an increase in cases was reported in the Netherlands, the majority of which affected children younger than 5 years old. ${ }^{14}$ In Sweden, 74 EV-D68 confirmed cases, among which there was one fatal case and another 10 presenting with severe respiratory or neurological symptoms, were reported during August-September 2016. The majority of cases were, once again, young children under 5 years of age. ${ }^{15}$ Cases have been found in all ages according to surveillance reports, and the most affected age group has yet to be defined. An increase in EV-D68 was also observed in other European countries, such as France, Italy, the UK, and Portugal, with reports of some cases being associated with AFP. ${ }^{16}$ A recent review has shown a causal relationship between EV-D68 and acute flaccid myelitis (AFM), supported by the application of Bradford Hill criteria. ${ }^{17}$

\section{EV-D68 classification and pathogenesis}

EV-D68 is a small, nonenveloped virus with a single stranded, positive-sense RNA genome of about 7.5 kilobases, which includes a single open reading frame which encodes four structural proteins (VP1 to VP4) and seven nonstructural proteins ( $2 \mathrm{~A}$ to $2 \mathrm{C}$ and $3 \mathrm{~A}$ to $3 \mathrm{D}$ ), a $5^{\prime}$ untranslated region (UTR) with a hairpin-loop secondary structure, and a $3^{\prime}$ UTR with a poly(A) $\operatorname{tract}^{18}$ (Figure 1). Based on the VP1 nucleotide sequence, EV-D68 strains have been classified into three distinct genetic clades; A, $\mathrm{B}$, and C. ${ }^{18}$ These are further divided into subclades A1 and A2, B1 and B2, and, since the 2014 outbreaks, two more subclades were described, B3 and D. ${ }^{14,15,19-21}$ In 2014, the predominant circulating EV-D68 subclade was B1, 6,20 and in 2016, the new subclade B3. ${ }^{14,15}$ EV-D68 belongs to the family Picornaviridae and genus EV, which comprises 12 species designated $\mathrm{EV} A$ to $\mathrm{H}$ and $\mathrm{J}$ and $\mathrm{RV} A$ to $\mathrm{C}{ }^{22}$ EV-D68 belongs to the EV D species, along with EV-D70, associated with acute hemorrhagic conjunctivitis; EV-D94, causative agent of AFP; EV-D111 and EV-D120, identified in nonhuman primates. ${ }^{18} \mathrm{EV}-\mathrm{D} 68$ is biologically similar to $\mathrm{RV}$, in that it is mainly associated with respiratory disease, and was previously named RV87. However, it was not until recently that severe cases of respiratory disease due to EV-D68 were reported. ${ }^{3,9,10,23}$

In general, EV infect via the fecal-oral route and are normally transmitted by inhalation of aerosolized viruscontaining material. However, manual transmission to the airway from environmental surfaces also occurs. After an incubation period of 1-2 weeks, symptoms may develop. During the first week of illness, the virus can generally be found in the nasopharynx, where it attaches to sialic acid

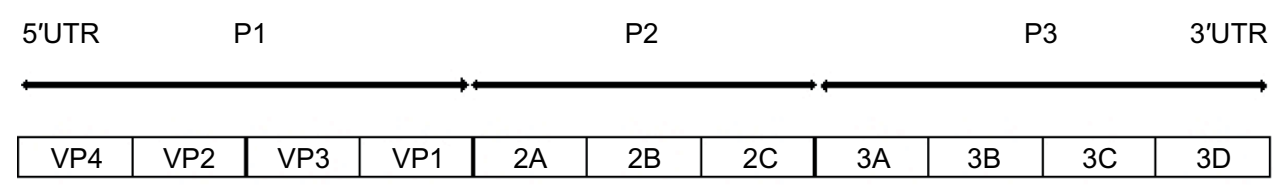

Figure I Schematic presentation of the genomic structure of enterovirus D68. 
(SA) receptors in the epithelial cells of the upper respiratory tract (URT) mucosa. EV-D68 spreads locally and can enter the bloodstream to gain access to organs such as meninges and other parts of the central nervous system. EV-D68 has been shown to have a higher affinity for $\alpha-2-6$-linked SA than $\alpha$-2-3-linked SA receptors as is the case of other respiratory viruses. The ability of the virus to bind to $\alpha-2-3$-linked SA receptors is an observation that supports that the virus can cause infections in the lower respiratory tract (LRT). Animal models have demonstrated that the virus can spread from URT to LRT after nasal infection and cause symptoms in the lungs. ${ }^{24}$

\section{Clinical presentation}

EV-D68 was first detected in cases presenting with respiratory symptoms like rhinorrhoea, sneezing, coughing, or sore throat combined with fever and malaise. EV-D68 can cause both URT and LRT infection and has been associated with bronchitis, bronchiolitis, wheezing illness, and exacerbation of asthma in children. ${ }^{18,25-27}$ Fatal respiratory infections with EV-D68 have been reported both in children and in adults, particularly in patients with comorbidities or immunosuppression. ${ }^{18,28}$ In addition, EV-D68 has been found in cases suffering from various neurological diseases. A fatal outcome has also been described in a meningomyeloencephalitis patient with EV-D68 positive cerebrospinal fluid (CSF). ${ }^{29}$

Outbreak investigations of children with polio-like disease presenting with AFP or AFM have shown that EV-D68 can be found in respiratory specimens from a considerable number of cases, particularly those with severe infection. ${ }^{6,30-35}$ Many cases commonly show prodromal symptoms, such as fever from URT infections, and usually improve before development of paralysis, which, if it occurs, is generally observed within a week's time. Other symptoms, such as headache, stiff neck, and pain in the back and affected limbs, are commonly observed as URT symptoms decrease. A common feature among these cases is that the virus is not detected in CSF samples or stool specimens at onset of paralysis, possibly due to late or insufficient sampling. A recent review suggests that EV-D68 may act as a trigger to AFM and describes an average time period of around 4 days until maximum muscle impairment is reached, involving decreased or absent reflexes. ${ }^{31}$ In that study, an asymmetric distribution of paralysis was observed, with mostly upper limbs affected, and general motor function was still impaired a year later upon follow-up consultations. Other clinical manifestations included bowel or bladder dysfunction. Asthma was one of the most common comorbidities among the cases. ${ }^{31}$

\section{EV-D68 diagnostics}

EV-D68 sample collection should be performed according to clinical manifestations and preferably within the first days following symptom onset. Specimens include CSF, stool, respiratory specimens, eg, nasopharyngeal aspirates, nasopharyngeal swabs, broncho alveolar lavage, and blood, in severe cases. ${ }^{36}$ Serum collection for serological testing of EV-D68 is not a common practice in routine diagnostics. The need for sampling is associated with severity of the clinical presentation, and collecting multiple samples from different sites is important for increasing the chances of successful detection of EV-D68. Stool sample collection from patients with AFP is fundamental to exclude poliovirus. Respiratory specimens should also be included for analysis, as this virus is rarely detected in CSF and stool, and a negative result in these types of specimens does not rule out EV-D68 infection. Blood samples should also be considered, as EV-D68 viremia may, in some cases, be higher in blood than in the CSF.

Diagnostic testing for EV relies on molecular methods such as PCR targeting the $5^{\prime}$ UTR of the genome. This region presents high similarity between EV and RV and as such may in some cases result in false negative or false RV positives. Furthermore, due to high genetic similarity among the different EV types, this methodology does not allow for a reliable differentiation between EV-D68, RV, and other EV, such as coxsackie virus and echovirus, and as such should not be used as the preferred method for EV type discrimination. The general strategy for EV-D68 confirmation has been to perform pan-EV PCR on the UTR and/or the VP1 or VP2 followed by sequencing of the PCR products. ${ }^{37,38}$ However, this procedure is time-consuming, costly, and unfeasible for many diagnostic laboratories.

Food and Drug Administration-cleared molecular assays are currently available for detection of EV and RV in clinical samples through respiratory panel multiplex assays. An EVD68-specific RT-PCR method was quickly developed by the CDC following the outbreak in 2014 and was approved by the Food and Drug Administration under emergency use authorization only. ${ }^{39-41}$ Other RT-PCR methods, with different degrees of specificity and sensitivity, have also been developed ${ }^{42-44}$ as well as a duplex-assay for simulations screening and differentiation between non-D68 EV positive and $\mathrm{D} 68$ positive virus. ${ }^{45}$

Complete viral genomes of EV-D68 have been generated by high-throughput sequencing of overlapping amplicons from primary specimens, allowing for identification of new clades as well as by other whole-genome sequencing strategies. ${ }^{19,46-49}$ In addition, a "Fernon" reference strain has been 
provided by the French National Enterovirus Reference Laboratory to allow laboratories to test the accuracy of EV and EV/RV assays. EV-D68 viruses have also been included in Quality Control for Molecular Diagnostics External Quality Assessment panels.

An evaluation of the EV diagnostic analysis performed in Europe in 2014 has shown that many commercial available diagnostic assays do not discriminate adequately between $\mathrm{RV}$ and EV and/or do not discriminate EV-D68 from other $\mathrm{EV}^{43,50,51}$ In both the Europe and the USA, few clinical diagnostic laboratories employ systematic EV detection followed by genotyping in the routine screening strategy of respiratory materials. As EV-D68 is known to cross-react with RV and EV targets, it is recommended to do EV-D68 confirmation by sequencing or to perform at least two assays with different target regions for detection. The VP1 region has been the preferred region for EV-D68 genotyping.

\section{Antiviral treatment and vaccines}

Currently, there is no specific antiviral therapy or vaccine available for EV-D68 infections. Mild disease cases might benefit from supportive therapies but are otherwise selflimiting. In the case of severe LRT disease, supplemental oxygen, bronchodilators, systemic corticosteroids, and ventilator support are the main interventions used..$^{52}$ Treatment for AFM is empiric and often includes plasmapheresis and high-dose corticosteroids, aimed at reducing immune responses and inflammation or human intravenous immunoglobulin (hIVG) with potential immunomodulatory or antiviral effects. ${ }^{26}$ The rare and sporadic occurrence of the disease makes randomized control trials for investigating treatment effects difficult. Lack of suitable animal models has further hampered a more comprehensive understanding of the EV-D68 pathogenesis, therapy, and vaccine development. Recent studies in experimental mouse models indicate that immunomodulatory strategies, such as use of human intravenous immunoglobulin containing high titers of antiEV-D68 or vaccination, might be efficient for prevention and treatment of EV-D68-mediatied neurological disease, while corticosteroids should be used with caution. ${ }^{53,54}$ Moreover, studies of three potential therapies against EV-D68 in experimental mouse models suggest that the primary target of treatment should be the virus itself, rather than reducing the immune response. ${ }^{53,54}$

Several direct-acting EV inhibitors have been developed, including capsid binders and inhibitors of viral enzymes required for genome replication such as protease inhibitors, $3 \mathrm{D}^{\text {pol }}$ inhibitors, and $2 \mathrm{C}^{\text {ATPase }}$ inhibitors. ${ }^{55}$ So far, none have been approved for clinical use. The most widely studied compounds are capsid binders, of which pleconaril, vapendavir, and pocavir are undergoing clinical trials. ${ }^{55}$ However, a disadvantage of capsid binders is the emergence of resistant EV-D68 variants, which complicates their clinical use. ${ }^{56,57}$ Protease inhibitors may be more promising as direct-acting antiviral drugs, particularly since these often have broadspectrum anti-enteroviral effects. Rupintrivir is the most studied among the protease inhibitors tested against EV and has shown broad-acting in vitro effects. However, clinical development was halted due to poor oral availability and lack of effect in naturally infected patients in clinical studies. Nevertheless, several rupintrivir derivatives are under development as well as preclinical studies of nonpeptidomimetic small molecule inhibitors with a better bioavailability than rupintrivir. ${ }^{55,56} 3 \mathrm{D}^{\mathrm{pol}}$ inhibitors such as ribavirin have shown promising results for some viruses; however, few have yet been developed against $\mathrm{EV}^{55}$ While several $2 \mathrm{C}^{\text {ATPase }}$ inhibitors have been identified, their effect against $\mathrm{EV}$ remains to be tested in clinical trials. Drug repurposing studies of $2 \mathrm{C}^{\text {ATPase }}$ inhibitors identified fluoxetine (ie, Prozac ${ }^{\circledR}$ ) as a potential drug for EV, and it was shown to reduce EV-D68 growth in vitro. However, adding to issues related to dosage and side effects, a recent study found no effect on EV-D68 viral loads or symptoms in an experimental mouse model..$^{53,54,58}$ Finally, inhibitors of host factors involved in EV infection might be used in treatment. These may have broad-spectrum antiviral activity but are also associated with drawbacks such as adverse effects and toxicity in the patient. ${ }^{55}$

The challenges of developing specific antiviral therapies against EV-D68 emphasize the need for vaccine development. Recent establishment of a suitable neonatal mouse model for EV-D68 disease, ${ }^{53}$ and identification of the cotton rat as a potential powerful animal model for natural EV-D68 infection, ${ }^{59}$ hopefully might accelerate future vaccine development.

\section{EV-D68 surveillance and outbreak management}

As a result of the EV-D68 outbreaks in 2014, many countries implemented measures to strengthen their national EV surveillance systems, such as enhanced screening of severe respiratory infections, according to well-defined case definitions, specific EV-D68 diagnostic testing, dissemination of information among healthcare providers and the general public, and enhanced screening of AFP cases. Ongoing surveillance is key for timely detection and effective response to outbreaks and to ensure supportive 
care. This surveillance, added to the mounting evidence of the AFP and EV-D68 association, as well as the cyclical EV-D68 pattern of 2-4 years during summer-autumn seasons, makes clinicians and laboratory staff evermore prepared to recognize and respond to potential EV-D68 outbreaks. An effective response to an outbreak requires coordination and clear communication among responders in multidisciplinary teams, including clinicians, nurses, infection prevention control officers, laboratory staff, epidemiologists, and public health officials, among others. A systematic and flexible outbreak investigation is essential to ensure the ultimate welfare of patients, by attending to all steps in the outbreak response process. These steps span from the early recognition of EV-D68 infection symptoms by the clinician who triggers the alert or by laboratory personnel detecting an increase in EV-D68 positive results, followed by the subsequent steps of an outbreak investigation, i.e., developing hypotheses, searching and describing further cases, implementing control measures, which should be done as early into the investigation as possible, to communication of final results. Given that, in cases of severe EV-D68 infection, the timeframe between disease onset and development of critical neurological symptoms is extremely short, usually spanning only a couple of days, the initial recognition and treatment stages are vital. Continued surveillance is essential to monitor EV-D68 incidence, circulating molecular strains as well as spectrum of illness and potential changes in patterns of illness severity.

\section{Concluding remarks}

Since its discovery in 1962, EV-D68 has made sporadic appearances until a series of outbreaks in 2014 caught worldwide public health attention. As a result, many countries strengthened their EV surveillance systems, to include also specific EV-D68 screening, with a particular focus on AFP and AFM cases. The implementation of VP1 and VP2 sequencing strategies, an extended EV surveillance including molecular epidemiology of both circulating and outbreak strains and a comprehensive viral characterization, would be an invaluable public health addition for EV reference laboratories. The underlying mechanisms for the apparent association between AFP and EV-D68 are still unclear, and merit further investigation as the number of reports suggesting this association increases. In addition, further studies are needed addressing the underlying host and viral factors, associated with severity of disease, as well as development of vaccines, in order to circumvent the current therapeutic challenges.

\section{Disclosure}

The authors report no conflicts of interest in this work.

\section{References}

1. Schieble JH, Fox VL, Lennette EH. A probable new human picornavirus associated with respiratory diseases. Am J Epidemiol. 1967;85:297-310.

2. Pastula DM, Aliabadi N, Haynes AK, et al; Centers for Disease Control and Prevention (CDC). Acute neurologic illness of unknown etiology in children-Colorado, August-September 2014. MMWR Morb Mortal Wkly Rep. 2014;63(40):901-902.

3. Meijer A, van der Sanden S, Snijders BE, et al. Emergence and epidemic occurrence of enterovirus 68 respiratory infections in The Netherlands in 2010. Virology. 2012;423:49-57.

4. Centers for Disease Control and Prevention. Clusters of Acute Respiratory illness associated with human enterovirus 68- Asia, Europe, and United States, 2008-2010. MMWR Morb Mortal Wkly Rep. 2011;60:1301-1304

5. European Centre for Disease Prevention and Control. Rapid Risk Assessment-Enterovirus Detected in the USA, Canada and Europe. Second update, 25 November 2014. Stockholm: ECDC; 2014.

6. Greninger AL, Naccache SN, Messacar K, et al. A novel outbreak enterovirus D68 strain associated with acute flaccid myelitis cases in the USA (201214): a retrospective cohort study. Lancet Infect Dis. 2015;15(6):671-682.

7. Lang M, Mirand A, Savy N, et al. Acute flaccid paralysis following enterovirus D68 associated pneumonia, France, 2014. Euro Surveill. 2014;19.

8. Pfeiffer HC, Bragstad K, Skram MK, et al. Two cases of acute severe flaccid myelitis associated with enterovirus D68 infection in children, Norway, autumn 2014. Euro Surveill. 2015;20:1-5.

9. Royston L, Tapparel C. Rhinoviruses and respiratory enteroviruses: not as simple as ABC. Viruses. 2016;8(1).

10. Ikeda T, Mizuta K, Abiko C, et al. Acute respiratory infections due to enterovirus 68 in Yamagata, Japan between 2005 and 2010. Microbiol Immunol. 2012;56:139-143.

11. Meijer A, Benschop KS, Donker GA, van der Avoort HG. Continued seasonal circulation of enterovirus D68 in the Netherlands, 2011-2014. Euro Surveill. 2014;19(42):pii 20935.

12. Pariani E, Pellegrinelli L, Merlone AD, Piralla A, Baldanti F, Binda S. Letter to the editor: need for a European network for enterovirus D68 surveillance after detections of EV-D68 of the new B3 lineage in Sweden and Italy, 2016. Euro Surveill. 2017;22(2):pii 30440.

13. Wang G, Zhuge J, Huang W, et al. Enterovirus subclade B3 strain circulating and causing an outbreak in the United States in 2016. Sci Rep. 2017;7:1242.

14. Knoester M, Schölvinck EH, Poelman R, et al. Upsurge of enterovirus D68, the Netherlands, 2016. Emerg Infect Dis. 2017;23(1):140-143.

15. Dyrdak R, Grabbe M, Hammas B, et al. Outbreak of enterovirus D68 of the new B3 lineage in Stockholm, Sweden, August to September 2016. Euro Surveill. 2016;21(46):pii 30403.

16. European Centre for Disease Prevention and Control. Rapid Risk Assessment: Enterovirus Detections Associated with Severe Neurological Symptoms in Children and Adults in European Countries, 8th August 2016. Stockholm: ECDC; 2016.

17. Dyda A, Stelzer-Braid S, Adam D, Chughtai AA, MacIntyre CR. The association between acute flaccid myelitis (AFM) and Enterovirus D68 (EV-D68) — what is the evidence for causation? Separator commenting unavailable. Euro Surveill. 2018;23(3): pii 17-00310.

18. Imamura T, Oshitani H. Global reemergence of enterovirus D68 as an important pathogen for acute respiratory infections. Rev Med Virol. 2015;25(2):102-114.

19. Huang W, Wang G, Zhuge J, Nolan SM, Dimitrova N, Fallon JT. Wholegenome sequence analysis reveals the Enterovirus D68 isolates during the United States 2014 outbreak mainly belong to a novel clade. Sci Rep. 2015;5:15223.

20. Brown BA, Nix WA, Sheth M, Frace M, Oberste MS. Seven strains of Enterovirus D68 detected in the United States during the 2014 severe respiratory disease outbreak. Genome Announc. 2014;2(6):e01201-14. 
21. Du J, Zheng B, Zheng W, et al. Analysis of enterovirus 68 strains from the 2014 North American outbreak reveals a new clade, indicating viral evolution. PLoS One. 2015;10(12):e0144208.

22. Principi N, Esposito S. Enterovirus D-68: an emerging cause of infection. Expert Rev Respir Med. 2015;9(6):711-719.

23. Imamura T, Fuji N, Suzuki A, et al. Enterovirus 68 among children with severe acute respiratory infection, the Philippines. Emerg Infect Dis. 2011;17:1430-1435.

24. Zheng H-W, Sun M, Guo L, et al. Nasal infection of enterovirus D68 leading to lower respiratory tract pathogenesis in ferrets (Mustela putorius furo). Viruses. 2017;9:E104.

25. Midgley CM, Jackson MA, Selvarangan R, et al. Severe respiratory illness associated with enterovirus D68-Missouri and Illinois, 2014. MMWR Morb Mortal Wkly Rep. 2014;63(36):798-799.

26. Esposito S, Bosis S, Niesters H, Principi N. Enterovirus D68 infection. Viruses. 2015;7(11):6043-6050.

27. Esposito S, Zampiero A, Ruggiero L, Madini B, Niesters H, Principi N. Enterovirus D68-associated community-acquired pneumonia in children living in Milan, Italy. J Clin Virol. 2015;68:94-96.

28. Bosis S, Esposito S. Enterovirus D68-associated community-acquired pneumonia in the pediatric age group. Curr Infect Dis Rep. 2017;19:12.

29. Kreuter JD, Barnes A, McCarthy JE, et al. A fatal central nervous system enterovirus 68 infection. Arch Pathol Lab Med. 2011;135: 793-796.

30. Van Haren $K$, Ayscue P, Waubant E, et al. Acute flaccid myelitis of unknown etiology in California, 2012-2015. JAMA. 2015;314(24):2663-2671.

31. Messacar K, Schreiner TL, van Haren K, et al. Acute flaccid myelitis: a clinical review of US cases 2012-2015. Ann Neurol. 2016;80(3): 326-338.

32. Sejvar JJ, Lopez AS, Cortese MM, et al. Acute flaccid myelitis in the United States, August-December 2014: results of nationwide surveillance. Clin Infect Dis. 2016;63(6):737-745.

33. Aliabadi N, Messacar K, Pastul DM, et al. Enterovirus D68 infection in children with acute flaccid myelitis, Colorado, USA, 2014. Emerg Infect Dis. 2016;22(8):1387-1394.

34. Poelman R, Schuffenecker I, Van Leer-Buter C, et al. European surveillance for enterovirus D68 during the emerging North-American outbreak in 2014. J Clin Virol. 2015;71:1-9.

35. Williams CJ, Thomas RH, Pickersgill TP, et al. Cluster of atypical adult Guillain-Barré syndrome temporally associated with neurological illness due to EV-D68 in children, South Wales, United Kingdom, October 2015 to January 2016. Euro Surveill. 2016;21(4):pii 30119.

36. Esposito S, Lunghi G, Zampiero A, et al. Enterovirus-D68 in the cerebrospinal fluid of two children with aseptic meningitis. Ped Infect Dis J. 2016;35(5):589-591.

37. Nix WA, Oberste MS, Pallansch MA. Sensitive, seminested PCR amplification of VP1 sequences for direct identification of all enterovirus serotypes from original clinical specimens. J Clin Microbiol. 2006;44(8):2698-2704.

38. Brittain-Long R, Nord S, Olofsson S, Westin J, Anderson LM, Lindh M. Multiplex real-time PCR for detection of respiratory tract infections. J Clin Virol. 2008;41(1):53-56.

39. Centres for Disease Control and Prevention. Enterovirus D68 (EVD68) 2014 Outbreak Strain-Specific Real-Time Reverse Transcription/ Polymerase Chain Reaction (rRT-PCR) Assay Instructions-version 10/14/20. CDC; 2014.

40. Centres for Disease Control and Prevention. Enterovirus D68 2014 RealTime RT-PCR Assay. 2015. Available from: https://www.fda.gov/downloads/MedicalDevices/Safety/EmergencySituations/UCM446784.pdf. Accessed January 31, 2018.
41. Zhuge J, Vail E, Bush JL, et al. Evaluation of a real-time reverse transcription-PCR assay for detection of enterovirus D68 in clinical samples from an outbreak in New York State in 2014. J Clin Microbiol. 2015;53(6):1915-1920.

42. Piralla A, Girelloa A, Premolia M, Baldanti F. A new real-time reverse transcription-PCR assay for detection of human enterovirus 68 in respiratory samples. J Clin Microbiol. 2015;53(5):1725-1726.

43. Poelman R, Schölvinck EH, Borger R, Niesters HG, van Leer-Buter C. The emergence of enterovirus D68 in a Dutch University Medical Center and the necessity for routinely screening for respiratory viruses. J Clin Virol. 2015;62:1-5.

44. Wylie TN, Wylie KM, Buller RS, Cannella M, Storch GA. Development and evaluation of an enterovirus D68 real-time reverse transcriptase PCR assay. J Clin Microbiol. 2015;53(8):2641-2647.

45. Bragstad K, Jakobsen K, Rojahn AE, et al. High frequency of enterovirus D68 in children hospitalised with respiratory illness in Norway, autumn 2014. Influenza Other Respir Viruses. 2015;9:59-63.

46. Tan Y, Hassan F, Schuster JE, et al. Molecular evolution and intraclade recombination of enterovirus D68 during the 2014 outbreak in the United States. J Virol. 2015;90:1997-2007.

47. Huang $\mathrm{W}$, Wang $\mathrm{G}$, Lin $\mathrm{H}$, et al. Assessing next-generation sequencing and bioinformatics tools for detection of enterovirus D68 and other respiratory viruses in clinical samples. Diagn Microbiol Infect Dis. 2016;85(1):26-29.

48. Chen L, Shi L, Yang H, et al. Identification and whole-genome sequencing of four enterovirus D68 strains in Southern China in late 2015. Genome Announc. 2016;4(5): $01014-16$.

49. Gong YN, Yang SL, Shih SR, et al. Molecular evolution and the global reemergence of enterovirus D68 by genome-wide analysis. Medicine. 2016; 95(31):e4416.

50. McAllister SC, Schleiss MR, Arbefeville S, et al. Epidemic 2014 enterovirus D68 cross-reacts with human rhinovirus on a respiratory molecular diagnostic platform. PLoS One. 2015;10(3):e0118529.

51. Shibib DR, Matushek SM, Beavis KG, Gawel SH, Charnot-Katsikas A. BioFire filmarray respiratory panel for detection of enterovirus D68. J Clin Microbiol. 2016;54(2):457-459.

52. Oermann CM, Schuster JE, Conners GP, Newland JG, Selvarangan R, Jackson MA. Enterovirus d68. A focused review and clinical highlights from the 2014 U.S. outbreak. Ann Am Thorac Soc. 2015;12(5):775-781.

53. Hixon AM, Yu G, Leser JS, et al. A mouse model of paralytic mielitis caused by eneterovirus D68. PLoS Pathog. 2017;13(2):e1006199.

54. Hixon AM, Clarke P, Tyler KL. Evaluating treatment efficacy in a mouse model of enterovirus D68 paralytic myelitis. J Infect Dis. 2017;216(10):1245-1253.

55. Bauer L, Lyoo H, van der Schaar HM, Strating JR, van Kuppeveld FJ. Direct-acting antivirals and host-targeting strategies to combat enterovirus infections. Curr Opin Virol. 2017;24:1-8.

56. Van der Linden L, Wolthers KC, van Kuppeveld FJ. Replication and inhibitors of enteroviruses and parechoviruses. Viruses. 2015;7(8):4529-4562.

57. Sun L, Meijer A, Froeyen M, et al. Antiviral activity of broad-spectrum and enterovirus-specific inhibitors against clinical isolates of enterovirus D68. Antimicrob Agents Chemother. 2015;59(12):7782-7785.

58. Ulferts R, van der Linden L, Thibaut HJ, et al. Selective serotonin reuptake inhibitor fluoxetine inhibits replication of human enteroviruses $\mathrm{B}$ and D by targeting viral protein 2C. Antimicrob Agents Chemother. 2013;57:1952-1956.

59. Patel MC, Wang W, Pletneva LM, et al. Enterovirus D-68 infection, prophylaxis, and vaccination in a novel permissive animal model, the Cotton Rat (Sigmodon hispidus). PLoS One. 2016;11(11):e0166336. 
Virus Adaptation and Treatment is an international, peer-reviewed open access journal focusing on the study of virology, viral adaptation and the development and use of antiviral drugs and vaccines to achieve improved outcomes in infection control and treatment. The journal welcomes original research, basic science, clinical \& epidemiological studies,
Dovepress

reviews \& evaluations, expert opinion and commentary, case reports and extended reports. The manuscript management system is completely online and includes a very quick and fair peer-review system, which is all easy to use. Visit http://www.dovepress.com/testimonials.php to read real quotes from published authors.

Submit your manuscript here: http://www.dovepress.com/virus-adaptation-and-treatment-journal 